\title{
Integration of RCM and PHM for the Next Generation of Aircraft
}

\author{
Alireza Ahmadi \\ Division of Operation and Maintenance \\ Engineering, \\ Luleå University of Technology, \\ SE-971 87 Luleå, Sweden. \\ +46920-493047 \\ alireza.ahmadi@1tu.se \\ Markus Klein \\ Saab Aerosystems \\ SE-581 88 Linköping, Sweden \\ +4613 - 184781 \\ markus.klein@saabgroup.com
}

\author{
Torbjörn Fransson \\ Saab Aerosystems \\ SE-581 88 Linköping, Sweden \\ +4613- 183248 \\ torbjorn.fransson@saabgroup.com
}

\author{
Anneli Crona \\ Saab Aerosystems \\ SE-581 88 Linköping, Sweden \\ +4613 - 180269 \\ anneli.crona@saabgroup.com
}

\begin{abstract}
With global cuts in defense budgets, air forces have to sustain the same level of readiness with a reduced number of aircraft. To succeed with this challenge, it is not sufficient to improve current maintenance concepts, but also new ones have to be introduced.

Traditionally, the development of on-board functions and maintenance concepts has been performed rather independently. The new approach is to focus on an integration of these two developments and to adapt a life cycle view together with a disregard of organizational boundaries.
\end{abstract}

To facilitate necessary changes, a study is performed by Saab and Luleå University of Technology. The study focuses on the possibilities to integrate RCM and PHM in a cost-effective way. The aim is to reduce the workload of maintenance development and to find out how to combine RCM and PHM to achieve a joint development of the aircraft and its maintenance program throughout the aircraft's whole life cycle.

The paper describes some similarities and differences between RCM and PHM. Furthermore, the paper describes some aspects of how RCM and PHM are complementing each other and what kind of adaptations that has to be done to achieve a successful integration. For example, a good application of RCM early in system design might generate a pull for PHM-technology integration and motivate design changes of the aircraft. Simultaneously, available PHMtechnologies might strongly affect the selection of applicable and effective maintenance tasks in the development of the initial maintenance program. PHM also gives a direct feedback of the maintenance program's performance. In addition, an approach for a stepwise integration of RCM and PHM is outlined. ${ }^{12}$

\section{TABLE OF CONTENTS}

1. INTRODUCTION 1

2. APPLICABILITY AND EFFECTIVENESS OF ON-

CONDITION MAINTENANCE TASKS.

3. INTRODUCTION TO PHM

4. OPERATIONAL AVAILABILITY AND IMPACT OF PHM.... 4 5. STRATEGY FOR STEPWISE INTEGRATION OF RCM AND

PHM

6. KEY ENABLERS .................................................................... 7

7. CONCLUSIONS AND DISCUSSION.............................................. 8

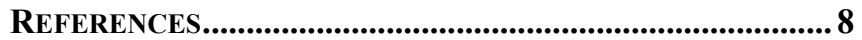

BIOGRAPHIES................................................................................. 9

\section{INTRODUCTION}

Reliability-Centered Maintenance (RCM) is a systematic methodology used to identify the maintenance tasks that are necessary to realize the inherent reliability of items at the lowest possible cost. Developing a scheduled maintenance program by means of RCM consists of identifying the maintenance strategies that are both applicable (technically feasible) and effective (worth doing).

On-Condition Maintenance (OCM) is one of the most important predictive maintenance strategies suggested by $\mathrm{RCM}$, which also has the top priority in the task-selection procedure. OCM is designed to detect a potential failure condition (and not functional failure state), so that action can be taken to prevent the functional failure or to avoid its consequences $[1,3,12]$. OCM discriminates between the items that require maintenance in order to prevent a func-

${ }^{1}$ 978-1-4244-2622-5/09/\$25.00 C2009 IEEE

${ }^{2}$ IEEEAC paper\#1366, Version 7 
tional failure, and those items that probably will meet the required performance and could survive to the next inspection.

In some cases, the time of the next inspection depends on the result of the previous inspection. If the inspection shows that the system is in an early stage of degradation, the next inspection interval could be adjusted [2], so that the degradation process is under control and the maximum Remaining Useful Life (RUL) could be utilized. Therefore, each serviceable item remains in operation and will be inspected or tested at regular intervals until its failure resistance falls below a defined level. This discrimination not only permits all items to realize most of their useful lives [12], but also reduces the maintenance load and downtime.

However, the item's capability to detect potential failures is the key to define an applicable OCM. If this detection capability is low, two alternatives are available. The first alternative is to abandon the OCM task in favor of some other maintenance tasks, i.e. restoration, discard, or failure finding. However, in many cases none of these alternative maintenance tasks are good solutions. Hence, the second alternative is to select a default action, i.e. run-to-failure (for non-safety consequences) or redesign. If run to failure is not favorable, redesign is the last alternative.

There are several alternatives for a redesign. One alternative is to increase the item's reliability performance (e.g., by increased strength or inclusion of redundancies), and consequently more costly. Another alternative is to improve the item's maintainability performance, i.e., to provide an item that is easy to access and restore and results in a short down-time. This could be done through the inclusion of some technologies, e.g. related to Prognostics \& Health Management (PHM). Consequently, maintainability is one of the main factors in achieving a high level of operational availability.

The prime objective in the development of a system, within constraints specified by operation and maintenance requirement, is cost-effectiveness. Consequently, there is always a trade-off between the degree of maintainability and related level of cost. As illustrated in Figure 1, system design attributes and system support elements impact both the technical and the economical sides of the cost-effectiveness relationship. In addition, a major projected Life Cycle Cost (LCC) for a system stems from the consequences of decisions made during the early design phases [6]. Those decisions pertain to the utilization of new technologies, selection of components and materials, selection of manufacturing process, incorporation of diagnostic routines, prognostics and maintenance support policies.

In this context, the designer must take into account the consequences of such interactions in order to achieve the predefined system effectiveness targets and minimize the cost.
Otherwise, the effectiveness will decrease and the cost will increase, since traditionally the performance element is usually emphasized in comparison with other design parameters, such as maintainability and maintenance support performance. [8]

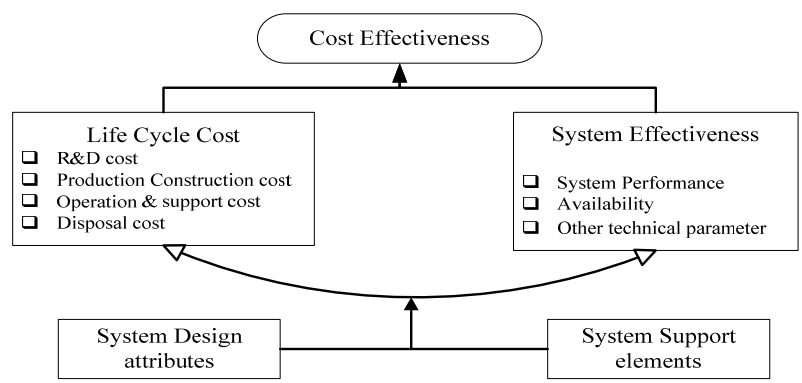

Figure 1 - The relationship between Life Cycle Cost (LCC) and system effectiveness to achieve cost effectiveness

When new and immature technologies are introduced, system upgrades will be required frequently as performance and Mean Time Between Failure (MTBF) improves. This also means that maintenance intervals might be escalated for upgrades, even if the maintenance tasks are the same. With a full RCM approach, there will be different, but fixed, maintenance intervals for each release. Hence, one major benefit of PHM would be an improved maintenance planning, even if the real effect of the upgrades is unknown at the service entry. Hence, the development of a maintenance program, which primarily is dimensioning the required maintenance support performance, should be done as early as possible in system design in order to influence necessary design changes of the technical system and in order to get the most applicable and cost-effective solution for system LCC.

To increase the system's availability performance in a costeffective way, it would be beneficial to integrate the RCManalysis in the design phase at proper time (not too late), so that it contributes in maintainability allocation in an effective way. This would lead to improved maintainability performance of the system through inclusion of new and innovative technologies for PHM.

\section{APPLICABILITY AND EFFECTIVENESS OF ON- CONDITION MAINTENANCE TASKS}

When designing to meet both economical requirements and competitive operation it soon becomes important to balance between what is technically feasible and what is economically acceptable. Thus, methodologies of engineering economy analysis should be used to provide results that will help to attain an acceptable balance. [9]

In order to achieve the above-mentioned requirements, the development of a scheduled maintenance program consists of determining which preventive task that are both appli- 
cable (technically feasible) and effective (worth doing). The applicability of a task depends on the reliability knowledge [7], failure characteristics of the item and solely on the type of the task; $[3,12]$.

Therefore, an applicable maintenance task must restore a system to its initial performance capability. In other words, a task is applicable if it eliminates a fault, reduces the probability of occurrence to an acceptable level or reduces the impact of failures. [7]

An on-condition inspection is applicable only if the failure characteristics of the item make it possible to define a potential failure condition in which detection of reduced failure resistance also would be possible. Moreover, a reasonably consistent and large enough time interval between the potential failure $(\mathrm{P})$ and the functional failure (F) must exist $[1,12]$. On the other hand, if the net P-F interval, or prognostic horizon associated with the minimum interval, is long enough for a suitable action to be taken to deal with the consequences of the failure, then the oncondition task is technically feasible. Otherwise, it is not possible to establish a meaningful task interval, and the task in question should be abandoned in favor of some other way of dealing with the failure. [1]

To develop an on-condition task, an identifiable and measurable degradation characteristic is required as an indicator of the item's resistance to failure. For a specific failure mode, it may be possible to define different kinds of degradation characteristics that can be used to reveal a potential failure condition. For example, several valid potential failure indicators for the failure mode "wear" could be specified as [10]:

- the amount of material lost through wear,

- the level of vibration induced by a worn segment of a rotating item, and

- the intensity of heat generated by friction associated with wear.

After deciding which degradation characteristic that is most suitable to use as an indicator, it is necessary to identify the P-F interval and the measurement methodologies. There are mainly five criteria, which an on-condition task must satisfy to be applicable $[1,12]$ :

1. it must be possible to detect increased failure probability for a specified engineering failure mode,

2. it must be possible to define a clear potential failure condition that can be detected by an explicit task,

3. there must be a reasonably consistent time interval between the potential failure and the functional failure (the P$\mathrm{F}$ interval),
4. it would be practical to monitor the item at an interval less than the P-F interval, and

5. the net P-F interval should be long enough to enable a proper action, to reduce or eliminate the consequences of the functional failure.

Normally, answering the applicability questions is quite clear-cut. This might be done through research, lab test or by trend analysis. However, deciding whether the task fulfills the objective in accordance with the effectiveness criteria is a more complex issue.

The effectiveness of a task is a measure of the result of the maintenance task objectives, which is dependant on the failure consequences [12]. In other words, the maintenance task's effectiveness is a measure of how well the task accomplishes the intended purpose and if it is worth doing [7]. The RCM logic classifies all failure consequences in the following four groups:

1. safety and environmental consequences,

2. operational consequences,

3. non-operational (economical) consequences, or

4. hidden failure consequences (including both safety and non-safety effects).

In general, the following criteria must be satisfied to make an on-condition task effective:

- Failures with safety/environmental consequences: the effectiveness criteria require that the task reduces the probability of critical failures to an acceptable level. In addition, it will be effective (worth doing) if it can be relied on to give enough warning of the failure to ensure that action can be taken in time to avoid the safety or environmental consequences. [1, 3]

- Failure with hidden safety consequences: an on-condition task intended to prevent a hidden failure should reduce the risk of multiple failures to an acceptably level. $[1,3]$

\section{INTRODUCTION TO PHM}

The concept of prognostics is to diagnose and predict Remaining Useful Life (RUL) of an item (e.g. device, component, or system). Systems with prognostic capability will be able to identify potential failures in advance and provide information on system health, and this information could be used to reduce unscheduled maintenance or to extend scheduled maintenance intervals [16, 17]. This prognostic capability offers tremendous advantages since the Mean Time To Repair (MTTR) for unscheduled 
maintenance is considerably larger than for scheduled maintenance [18].

Moreover, the information on impending errors and failures makes it possible to optimize the logistics for replacement of components, i.e. by pre-order of the device that is about to fail and prepare for personnel requirement. This can limit the spare-part inventory and further reduce the down-time of the system in question. Yet another benefit would be to adjust the operational profile of the aircraft, i.e., an aircraft with a certain potential failure could be used for a certain type of mission where the stress-factors for that particular failure are minimal.

A highly developed ability to use aircraft components and systems optimally is advantageous in order to achieve competitiveness on the aircraft market of today. This requires that degradations and impending faults are identified before they cause a failure and that the RUL can be estimated by using prognostic methodologies. The prognostic ability enables Condition-Based Maintenance (CBM) and offers the possibility to prolong RUL. By integrating on-board PHM technologies with off-board information system, it is possible to achieve Risk-Based Utilization \& Support (RBUS), where the current system's health are considered jointly with available support resources (e.g. personnel and spare parts). Altogether, this gives the operator an increased potential for improved aircraft availability, which can be exemplified by the concept of autonomic logistics within the Joint Strike Fighter (JSF) program.

The ability to predict when an impending fault will have a functional impact also has a positive effect on system safety, maintenance and Life Cycle Cost (LCC). These benefits could be achieved by (1) adapting operation to the actual system health, (2) reducing the need for unscheduled corrective maintenance since potential failures instead of functional failures are detected (i.e. transferring unscheduled maintenance to scheduled maintenance), (3) increasing the average useful life of Line Replaceable Units (LRUs) by estimating their RUL, i.e., by using the LRUs longer than advocated in the preventive maintenance program if the health condition of the unit permits it (i.e. transferring time-based maintenance to CBM), (4) preparing for maintenance activities before the aircraft has landed (e.g. reducing logistic and administrative delay times).

This goes well in hand with new contract models for Maintenance, Repair and Overhaul (MRO) that have emerged in the context of Contracting For Availability (CFA), e.g. Performance Based Logistics (PBL), Integrated Operational Support; Service-by-the-Hour, and Maintenance Free Operating Period (MFOP). These models enable a fixed-price total solution selling, i.e. the manufacturer does not only deliver the technical system and customer support, but now also has the end-to-end responsibility for the logistic supply chain [19].
Today, prognostic concepts and methodologies are developed and implemented for mechanical systems in different complex systems, where availability is the main concern, such as in military and aerospace. Examples of properties to consider in mechanical systems are: vibration, temperature, operating hours, and condition of fluids. [20]. These properties are relatively simple to monitor and collect data about. Moreover, the physics and models behind failure precursors for mechanical systems are well known. This provides a solid ground for PHM of mechanical systems.

Electronic systems, on the other hand, have traditionally not been subject to PHM since their time to wear-out has been longer than the life cycle of the whole system [21]. However, for very deep sub-micron processes this has changed as the circuit life-time for worst-case conditions are down to a few years or even a few months [22, 23]. With modern aircraft being increasingly dependent on avionics, the need for robust and accurate PHM systems will be increasingly important.

A trend in the aircraft industry is to introduce more electronics into the aircraft (More Electric Aircraft, MEA), exemplified by the Boeing B787 and Joint Strike Fighter (JSF), which are the first commercial and military aircraft respectively based upon MEA. The main objective of MEA is to reduce weight, in order to reduce the aircraft fuel consumption. This is realized by replacing mechanical systems by electronics, which in turn has raised demands of using prognostics on electrical components and circuit boards [24].

\section{OPERATIONAL AVAILABILITY AND IMPACT OF PHM}

The operational availability is the probability that a system or equipment, when used under stated conditions in an actual operational environment, will operate satisfactorily when called upon, see Equation 1.

$$
A_{O}=\frac{\frac{M T B M_{U} * M T B M_{S}}{M T B M_{U}+M T B M_{S}}}{\frac{M T B M_{U} * M T B M_{S}}{M T B M_{U}+M T B M_{S}}+\left(\frac{\lambda\left(\bar{M}_{c t}\right)+f(p t)\left(\bar{M}_{p t}\right)}{\lambda+f(p t)}\right)+M W T_{(A D T+L D T)}}
$$

In Equation 1, MTBM is the Mean Time Between Maintenance, where the index $U$ denotes unscheduled maintenance and the index $\mathrm{S}$ denotes scheduled maintenance. MWT is the Mean Waiting Time, which includes the Administrative Delay Time (ADT) and the Logistic Delay Time (LDT). The $\lambda$ denotes the failure rate and is connected to the mean time for corrective maintenance, while $f(p t)$ is the frequency of preventive maintenance, which is connected to the mean preventive maintenance time. 
The main time constituents of Equation 1 are illustrated in Figure 2, where also the expected impacts of PHM technologies are highlighted.

PHM technologies are expected to contribute to an increased uptime by eliminating unnecessary inspections. Hence, the MTBM is expected to be improved. This can be achieved through condition monitoring and redundancy management, which will allow continued operation, with or without any restrictions. However, the prognostic capability is also expected to enable an avoidance of unplanned maintenance and instead enable planned maintenance. In addition, this planned maintenance should as far as possible be corrective, so that only necessary maintenance are performed.
Hence, any scheduled maintenance that is based on time measures (e.g., flight hours, cycles, or calendar time) should be eliminated.

Decisions about operation can also be based on prognoses, in the same way as diagnoses, with the advantage of a planning horizon. Hence, prognostics also enable a control of the aging of technical systems. [4, 12] These possibilities will affect the MTBM positively. The prognoses enable also decisions about recommended maintenance (e.g. opportunistic maintenance). This last feature will also affect the MTBM positively. [4, 5, 11]

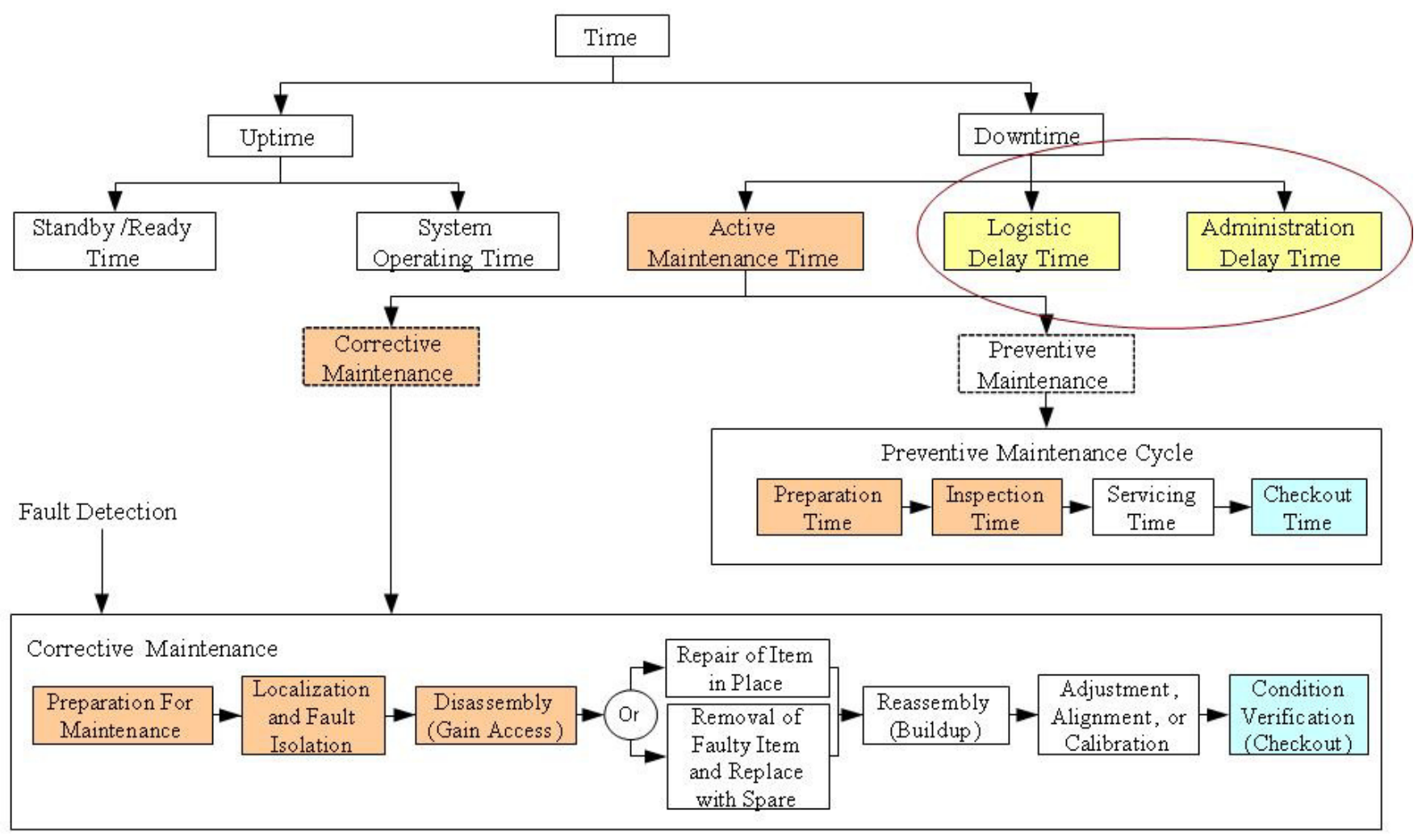

Figure 2 - Different time components that have impact on the operational availability 


\section{STRATEGY FOR STEPWISE INTEGRATION OF RCM AND PHM}

The RCM methodology is well established and incorporated into the processes at Saab. As this methodology is well known by customers and authorities, maintenance planning is easy to review and is seldom questioned.

By implementing PHM, the new concept has to be implemented, not only by the manufacturers and users, but also by customers and authorities. The responsibility to give sufficient evidence will then fall on the manufacturer, while the customers and authorities are free to request any evidence needed, and the burden of proof will be much higher than for methodologies already in practice.

The plan is therefore to implement PHM incrementally in two dimensions, i.e. increase the number of systems and refine the accuracy. First of all, the selection of which systems that PHM should be applied for, and secondly to decide the extent to which it should be implemented. A step-wise approach to implementation of prognostics render the possibility to gain early benefits from small technology achievements instead of waiting until all technologies are in place. Starting up in a small scale will give valuable feedback along the way.

A proposed way of working for integration of RCM and PHM in the early design phase is illustrated in Figure 3, which shows the strategy for how to select systems eligible for RCM and PHM. If cost-effective, a full PHM solution would be the final target. However, since there is a lack of experience data and knowledge of the physics of failures, well-established methodologies, such as RCM, would be used. For low-cost items with no or marginal failure consequences, run-to-failure will be the most likely option.

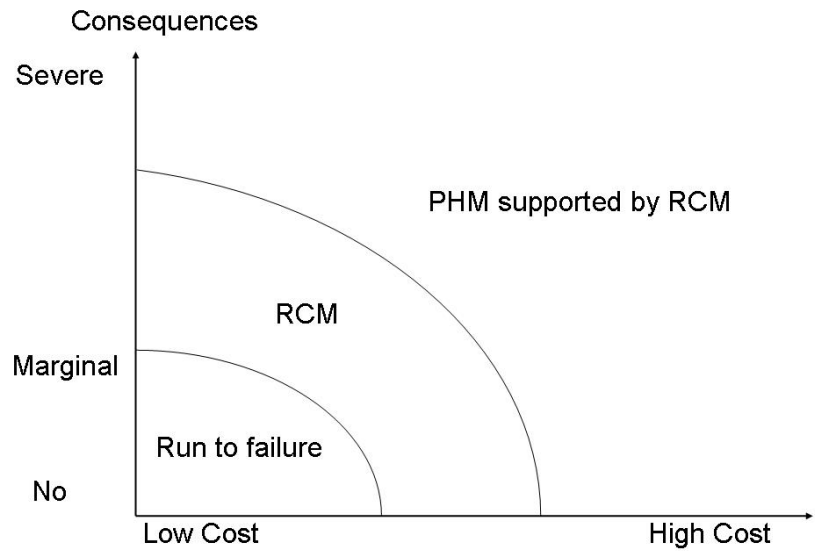

Figure 3 - Approach selection criteria
Assuming a successful integration of RCM-analysis in the design phase, there are mainly three approaches that may be applicable for taking care of the operational availability:

\section{Run-to-failure}

\section{RCM}

\section{PHM supported by RCM}

Out of these three maintenance approaches, the one that is most cost-effective should be selected.

Another selection criterion is based on the nature of the distribution of a failure, as illustrated in Figure 4. When the failure distribution is narrow, i.e. the variance is low, the MTBF can be well predicted using fleet data and the intervals for time-based maintenance can be set with a high confidence.

However if the variance is large, the occasion for timebased maintenance will either: 1) be set too conservatively resulting in much unused life of items, or 2) be set too late, resulting in extensive corrective maintenance. For the latter two scenarios it may be more cost-effective to incorporate information on the individual health of an item, as in PHM.

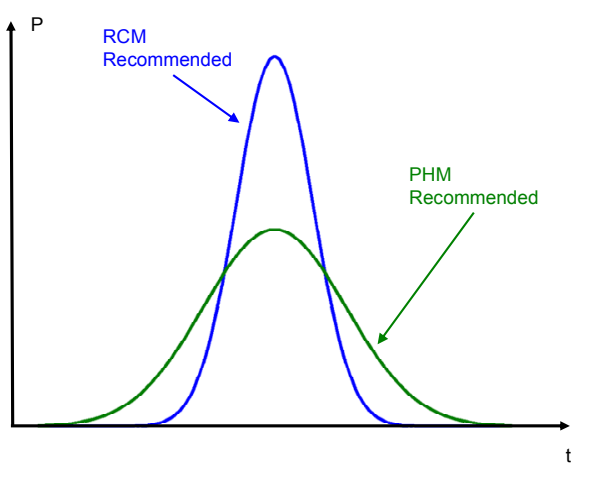

Figure 4 -Failure distribution

There are basically two major approaches to PHM [13]: data-driven models and physics-of-failure models.

In the data driven approach, mathematical models are used to calculate RUL based on statistical data. Even if a sufficient amount of data is available to ensure confidence, the uncertainty will be high operating on single individuals.

In the physics-of-failure approach, models that cover all aspects of physics of failure are used and the models are continuously compared with actual data. Until the reliability of the models is sufficient enough the optimal solution would be to combine data driven methodologies with physical models. 
Physics of failure models for avionics will still need more maturing before they have reached an acceptable TRL level. Current research within the Saab group in prognostics on avionics comprises prognostics of solder joint failures due to thermal fatigue [13] and monitoring of power supply currents [14].

Enhanced recording will contribute to more knowledge and the incremental development process will allow for a continuous upgrade of algorithms and parametric values in off-board support systems, but also in the on-board system using field loadable data.

The challenge now is to learn how to convert all recorded data into useful information and produce reliable maintenance reports. As user acceptance will rely much on the accuracy, immature prognostics could be very damaging for the confidence. At the same time, the use of prognostics has to be introduced in a small scale to gain field experience.-

There are two basic ways to handle prognostic based maintenance reports. The easiest way is to implement it in the Maintenance Ground Support System (MGSS). In MGSS, calculations can be done based on both recorded data downloaded from the aircraft, as well as on statistical and usage-based data from other units in the fleet. This methodology is recommended when the prognostic horizon is relatively long.

The second way is to have a real-time on-board monitoring system. If failures are predicted to occur within the next couple of days, an MGSS solution would be too slow. The way to operate then is to create a maintenance report in the on-board system. This will not result in any warning for the pilot, but the need for action will show in the Quick Report that is displayed after each mission.

The Quick Report (Figure 5), contains all information needed for the pilot to decide whether the aircraft can be used in a new mission, or if the aircraft shall be handed over to the maintenance organization. If no warnings occurred during the mission, it is most likely that a new mission could be started directly after turnaround. Maintenance actions can then be presented from the diagnostic system.

The diagnostic system includes logic for evaluation of maintenance report information, and as the diagnostic system can be field loadable, continuous upgrades of the function is possible without software changes and without costly re-certification.

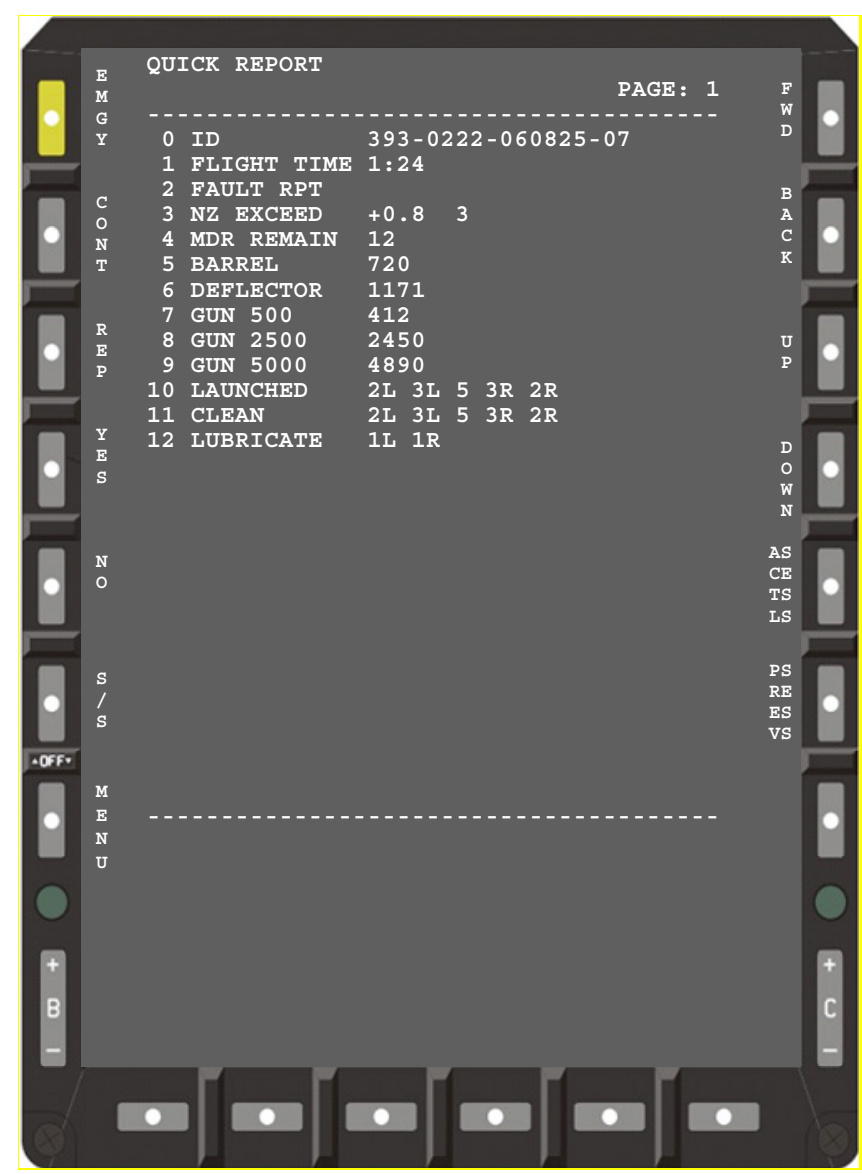

Figure 5 - Example of Quick Report in the Gripen Aircraft

\section{KEY ENABLERS}

Full PHM still looks like a distant vision. There is still much knowledge to be gathered.

- Physics of failure models for all systems including avionics.

- Accurate prediction methodologies. Awareness of the degree of uncertainty for each prediction is essential in order to take the correct decisions.

- An integrated feedback loop to bring field experience back to the designers in a more efficient way.

- Field loadable functions for diagnostics and prognostics. Delivered functions shall be easier to introduce and not require the same form of certification as software and other functions.

- An integrated process comprising seamless handovers between developers from both systems development and maintenance engineering. 


\section{CONCLUSIONS AND DiscuSSION}

Previously, the main focus has been on downtime. Better diagnostic capability and smarter maintenance will reduce time for both unscheduled as well as for scheduled maintenance. A shift to uptime focus requires a more proactive maintenance concept, where most of the maintenance planning and preparation is done during uptime and where prognostics will be one of the key enablers.

The Gripen on-board system and support systems are well prepared to cater for a continuous growth in functionality. The current and future architecture for information management supports stepwise upgrades of the material system, some planned a decade in advance, others to be planned and implemented on a much shorter notice.

Our current focus is to cater for a continuation of the Gripen maintenance concept, where PHM is added as an important corner-stone. A continuous growth of information and upgrades of models, their physical behavior, predictability and prognostic functionality will cater for a more efficient use of resources while at the same time focusing on the most efficient use of an aircraft or a fleet of aircraft.

\section{REFERENCES}

[1] J. Moubray (1997) Reliability-Centred Maintenance: RCM II, Second Edition, Butterworth Heinemann, Oxford.

[2] Welte, T; Vatn, J; Heggset, J., 2006, Markov State Model for Optimization of Maintenance and Renewal of Hydro Power Components, Proceedings PMAPS 2006, Stockholm, Sweden.

[3] MIL-STD-2173 Reliability Centered Maintenance Requirements for Naval Aircraft, Weapons Systems and Support Equipment.

[4] R.K. Mobley (1990) An Introduction to Predictive Maintenance, Van Nostrand Reinhold, New York.

[5] J.D. Campbell and A.K.S. Jardine (2001) Maintenance Excellence: Optimizing Equipment Life-Cycle Decisions, Marcel Dekker, New York.

[6] Blanchard, B.S., Verma, D., Pererson, E.L. (1995), "Maintainability: A key to effective serviceability and maintenance management", John Wiley and Sons, New York.

[7] Rausand, M., Van. J. 1998. Reliability Centered Maintenance. Risk Reliability in Marin Technology, Balkeman, Holland
[8] D’Addio, G.F., Firpo, P., Savio, S., (1998).Reliability Centered Maintenance: a solution to optimise mass transit system cost effectiveness. IEEE Int. Conference on Developments in Mass Transit Systems, London, UK, $211-216$.

[9] Sulivan, W.G., Wicks, E.M, Luxhoj, J., 2006. Engineering Economy. Thirteen ed. Pearson: New jersey

[10] NAVAIR 00-25-403, Guidelines for the Naval Aviation Reliability-Centered Maintenance Process, Patuxent River: Naval Air Systems Command, 2005.

[11] P. Söderholm and P.A. Akersten, Aerospace Diagnostics and Prognostics in a TQM-Perspective, in $15^{\text {th }}$ International Congress on Condition Monitoring and Diagnostic Engineering Management (2002) COMADEM International, Birmingham, UK. 80-89.

[12] F.S. Nowlan and H.F. Heap (1978) Reliability-Centered Maintenance, Department of Defence, Washington, DC.

[13] Hess A, Frith P, Calvello G, Engel S, Hoitsma D "Challenges, Issues, and Lessons Learned Chasing the Big 'P': Real Prognostics Part 2”, paper \# 1489, 2006 IEEE Conference, March 2006.

[14] J. Johansson, P. Leisner, Method for Prognostics of Thermal Fatigue Failure of Solder Joints in Avionic Equipment, IEEE PHM Conference October 2008.

[15] T. Sundström, B. Mesgarzadeh, M. Krysander, M. Klein, I. Söderquist, A. Crona, T. Fransson, A. Alvandpour, Prognostics of Electronic Systems through Power Supply Current Trends. IEEE PHM Conference October 2008.

[16] J. Gu, N. Vichare, T. Tracy, and M. Pecht, "Prognostic implementation methods for electronics," in Proc. 53rd Annual Reliability \& Maintainability Symposium (RAMS), 2007.

[17] N.M. Vichare and M.G. Pecht, "Prognostics and health management of electronics," IEEE Trans. on Components and Packaging Technologies, vol. 29, no. 1, March 2006.

[18] H. Hecht, "Why prognostics in avionics?," in Proc. IEEE Aerospace Conference, March 2006.

[19] P. Söderholm, "A system view of the no fault found phenomenon," J. Reliability Engineering and System Safety, 92(1), pp. 1-14, 2006. 
[20] D. Droste and K. Finklea, "Prognostics for electronic systems," in Proc. IEEE System Readiness Technology Conference, Sept. 2006.

[21] P. Sandborn “A decision support application for prognostic health management (PHM) approaches to electronic systems," in Proc. Reliability and Maintainability Symp., Jan. 2005.

[22] M. Ruberto, T. Maimon, Y. Shemesh, A.B. Desormeaux, Z. Weiquan Y. Chune-Sin, "Consideration of age degradation in the RF performance of CMOS radio chips for high volume manufacturing," in Radio Frequency integrated Circuits (RFIC) Symposium, pp. 549-552, June 2005.

[23] Circuit aging: a new phenomenon for SoC designs [Online]. Available: http://www.electronicdesign.com/Articles/Index.cfm?Art icleID $=4523$.

[24] S. Mishra S. Ganesan, M. Pecht, and J. Xie, "Life consumption monitoring for electronics prognostics," in Proc. IEEE Aerospace Conference, March 2004.

[25] International Electrotechnical Vocabulary (IEV) no 19202-05. Available at:

http://www.electropedia.org/iev/iev.nsf/display?openfor m\&ievref=191-02-05

\section{BIOGRAPHIES}

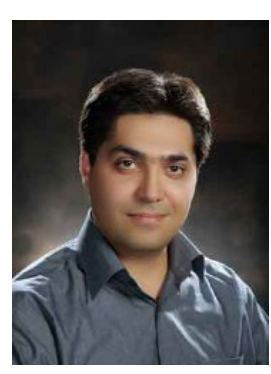

Alireza Ahmadi is a $\mathrm{PhD}$ candidate at the Division of Operation and Maintenance Engineering, Luleå University of Technology, Sweden. His research topic is related to the risk, reliability, availability, and costeffectiveness analysis for aircraft maintenance program development. He has more than 10 years experience in civil aviation maintenance, as Aircraft licensed Engineer, Project Manager and head of Production Planning and Control Department. He received his Licentiate degree in Operation and Maintenance Engineering 2007.

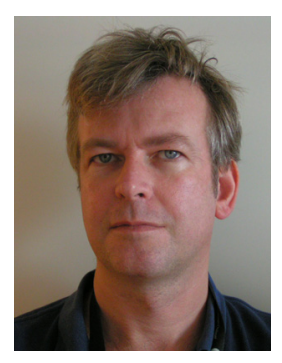

Torbjörn Fransson is a senior systems engineer at Saab Aerosystems, Linköping Sweden. He started his career at Saab in 1986 doing a study in how to use AI tools to plan process allocation in a real time system. From 1987 until 1999, he developed and led development of software for the Saab 39 Gripen fighter. He has also, as manager for a software quality and methods department lead the development and deployment of configuration management systems and introduction of new processes and methods. $\mathrm{He}$ is currently Technical Manager for Monitoring, Diagnostics, Prognostics and Recording functions. He received his Master of Science, Computer Technology, at the University in Linköping in 1988.

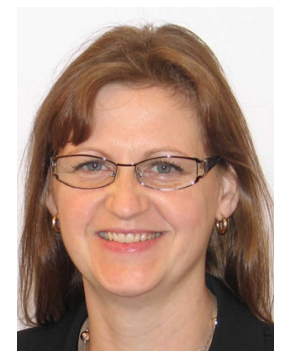

Anneli Crona is a senior systems engineer at Saab Aerosystems, Linköping Sweden. She is currently in charge of $\mathrm{R} \& \mathrm{D}$ programs within the monitoring and diagnostic area and leading the development of operational availability, maintenance, diagnostic and prognostic functions within the Gripen Next Generation project. Previously her choice of career has taken her through the pulp-and paper industry, the high-voltage power distribution industry and the telecommunication industry.

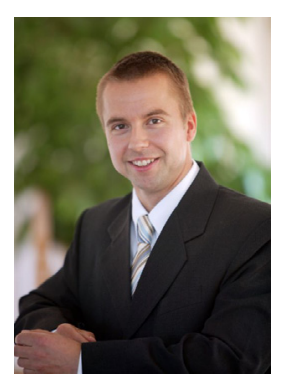

Markus Klein is a systems engineer at the division of Monitoring, Diagnostics, Prognostics and Maintenance Recording at Saab Aerosystems, Linköping, Sweden. He received his MSc degree in Applied Physics and Electrical Engineering in 1998 and $\mathrm{PhD}$ in Vehicular Systems in 2007, both from the University of Linköping, Sweden.

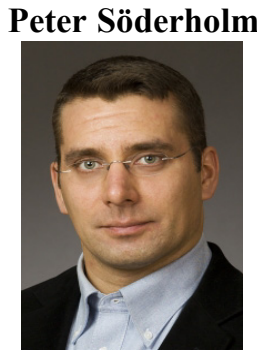

is Assistant Professor at Luleå University of Technology, the Division of Quality Technology, Environmental Management - and Social Informatics. His research topic is dependability with a focus on the application of Information \& Communication Technology (ICT), mainly within aviation. He received his MSc degree in 2001 in Mechanical Engineering and $\mathrm{PhD}$ in Operation and Maintenance Engineering in 2005, both from Luleå University of Technology, Luleå, Sweden. He is member of the International Electrotechnical Commission's (IEC) Swedish National Technical Committee 56 (TC 56: Dependability) 